\title{
ALVEOLAR TYPE II CELL TRANSPLANTATION RESTORES PULMONARY SURFACTANT PROTEIN LEVELS IN LUNG FIBROSIS
}

Raquel Guillamat-Prats ${ }^{1,2}$, Gemma Gay-Jordi ${ }^{1,2}$, Antoni Xaubet $^{2,3}$, Victor I Peinado ${ }^{2,3}$, Anna Serrano-Mollar ${ }^{1,2}$

${ }^{1}$ Department of Experimental Pathology, Institut d'Investigacions Biomèdiques de Barcelona; Consejo Superior de Investigaciones Científicas (IIBB-CSIC), Barcelona, Spain. Institut d'Investigacions Biomèdiques August $\mathrm{Pi}$ i Sunyer, Barcelona, Spain. ${ }^{2}$ Centro de investigaciones Biomédicas en Red de Enfermedades Respiratorias (CIBERES) Spain. ${ }^{3}$ Servei de Pneumologia; Institut Clínic del Tórax; Hospital Clínic, Barcelona, Spain.

Correspondence and requests for reprints should be addressed to:

Anna Serrano-Mollar

Dept. Experimental Pathology, IIBB-CSIC-IDIBAPS. C/ Rosselló, 161, 7ª, 08036, Barcelona, SPAIN

Phone: +34-93-3688307

FAX: +34-93-3638301

anna.serranomollar@iibb.csic.es

Running Title: Type II cells restore surfactant in lung fibrosis

Key words: Alveolar type II cells, cell therapy, pulmonary fibrosis, surfactant.

Word Count: 2534

Funding: This work was supported by Grant FIS PS09/02362 (Spanish Ministry of Health), by MTV3122410, by SEPAR (Sociedad Española de Neumología y Cirugía Torácica) and by FUCAP. Raquel Guillamat-Prats was supported by a Grant from CIBERES (Centro de investigaciones Biomédicas en Red de Enfermedades Respiratorias). 


\section{Abstract}

Rationale: Alveolar type II cell transplantation has been proposed as a cell therapy for the treatment of idiopathic pulmonary fibrosis. Its long-term benefits include the repair of lung fibrosis, but its success partly depends on the restoration of lung homeostasis.

Objectives: To evaluate surfactant protein restoration after alveolar type II cell transplantation in an experimental model of bleomycin-induced lung fibrosis in rats. Methods: Lung fibrosis was induced by intratracheal instillation of bleomycin. Alveolar type II cells were obtained from healthy animals and transplanted 14 days after bleomycin instillation. Furthermore, one group transplanted with alveolar macrophages and another treated with surfactant were established to evaluate the specificity of the alveolar type II cell transplantation. The animals were sacrificed at 21 days after bleomycin instillation. Lung fibrosis was confirmed by a histological study and an evaluation of the hydroxyproline content. Changes in surfactant proteins were evaluated by mRNA expression, Western blot and immunofluorescence studies.

Measurements and Main Results: The group with alveolar type II cell transplantation was the only one to show a reduction in the degree of lung fibrosis and a complete recovery to normal levels of surfactant proteins.

Conclusion: In conclusion, this study shows that one of the mechanisms involved in the beneficial effect of alveolar type II cell transplantation is the restoration of the lung surfactant protein levels, needed for proper respiratory function. 


\section{$\underline{\text { Introduction }}$}

Idiopathic pulmonary fibrosis (IPF) is a chronic, progressive and fatal disease of unknown etiology. The median survival time is 3 to 5 years from the time of diagnosis (1). At present there is no effective pharmacological therapy for IPF, although some new treatment approaches have shown a degree of efficacy (2-3).

It has been suggested that chronic injury and apoptosis of alveolar type II cells (ATII) underlie the development and progression of IPF (4-5), ultimately resulting in fibroblast proliferation, increased extra-cellular matrix deposition and loss of alveolar structure (6). In normal physiological conditions, the renewal of alveolar epithelial cells occurs through the specific proliferation and differentiation of ATII into alveolar type I cells (ATI). In pulmonary fibrosis, however, the ATII and ATI die, compromising the normal regeneration process and leading to an aberrant wound healing response (4-6). Therefore, any strategy seeking to attenuate or reverse pulmonary fibrosis should involve the maintenance of alveolar epithelium integrity. In this respect, our group has previously demonstrated that intratracheal transplantation of ATII is able to reverse bleomycin (BLM)-induced pulmonary fibrosis in a rat model (7).

ATII have a great number of complementary physiological functions, besides replacing alveolar epithelial cells (8). ATII are the only cells to synthesize, secrete and recycle the pulmonary surfactant needed to prevent alveolar collapse, by reducing the surface tension across the air-liquid interface of the alveoli (9). The presence of lung surfactant also helps to maintain the lung's gas exchange area and prevents the formation of edema. The loss of ATII during the development of pulmonary fibrosis contributes to alveolar collapse caused by a lack of surfactant (10).

Pulmonary surfactant is a complex molecule composed of $90 \%$ lipids and $10 \%$ proteins. There are four surfactant proteins (SP-A, SP-B, SP-C and SP-D) and they play pivotal roles in the alveolar homeostasis and host defence. 
Several studies have shown that changes in pulmonary surfactant could cause interstitial lung diseases in both children and adults (11-16). Moreover, patients with IPF have presented a down-regulation of SP-B and SP-C levels during the fibro-proliferative period (11-13). Mutations in the gene encoding SP-C have been identified in both familial and sporadic cases of IPF (15), while transgenic mice deficient in SP-C or SP-D have shown greater susceptibility to BLM, indicating that a lack of SP-C or SP-D predisposes to the development of lung fibrosis $(17,18)$. Lastly, patients with IPF have also presented alterations in the surfactant protein processing due to endoplasmic reticulum stress (19). All these findings show that surfactant alterations are involved in the development of pulmonary fibrosis and may be partly responsible for alveolar collapse, ventilation-perfusion mismatching and/or hypoxemia (20).

In this study we have focused on changes in pulmonary surfactant protein levels during BLMinduced lung fibrosis in rats and the ability of ATII transplantation to restore these surfactant protein levels.

\section{Methods}

Animals. Sprague-Dawley rats, weighting $200-225 \mathrm{~g}$ at the beginning of the experiment, were used, in accordance with the European Community (Directive 86/609/EEC) and Spanish guidelines for experimental animals and it was approved by the institutional committees of animal care and research of University of Barcelona.

Bleomycin-induced lung fibrosis. Lung fibrosis was induced by intratracheal instillation of a single dose of BLM (2.5 U/kg) (Sigma, USA) dissolved in $400 \mu$ of sterile saline under isofluorane anesthesia (7). Control animals received the same volume of saline. The animal body weights were recorded every 2 days during the course of the experiment.

Isolation of Alveolar Type II Cells. ATII were isolated from healthy donor animals. For more details, see the online data supplement. 
Cell yield, purity and characterization of freshly isolated ATII were established by the presence of intracellular alkaline phosphatase (Sigma, USA) (Fig. 1A), lamellar bodies and microvilli at the electron microscope level (Fig. 1B) by immnunofluorescence (Fig 1C), realtime PCR (Fig 1D) and by flow citomety (Fig 1E). For more details, see the online data supplement.

Isolation of Alveolar Macrophages. An additional alveolar macrophage (AM) transplantation group was established,using another unrelated cell type, to evaluate whether the beneficial effect was specific to ATII transplantation. AM were isolated from healthy donor animals. For more details, see the online data supplement.

Cell yield, purity and characterization of freshly isolated AM were evaluated in cytospin preparations, after staining with the Diff-Quick kit (Diagnostics Grifoll S.A, Spain). Over 95\% $(n=8)$ of brochoalveolar lavage cells were macrophages (Fig. 1F). AM were also characterized by transmission electron microscopy (Fig 1G).

Transplantation procedure. At day 14 after intratracheal BLM, recipient animals were transplanted with labelled ATII or labelled AM by intratracheal instillation $\left(2.5 \times 10^{6}\right.$ cells/animal suspended in $400 \mu$ l of sterile saline) under isofluorane anesthesia. The control group received the same dose of cells 14 days after saline instillation. The animals were sacrificed 21 days after the induction of lung fibrosis (7).

Surfactant administration. A surfactant administration group was also established in order to evaluate the specificity of the beneficial effect of ATII transplantation. At day 14 after intratracheal BLM, the animals were treated with commercial surfactant (Survanta, Abbott Laboratories, Spain) by intratracheal instillation $(0.5 \mathrm{ml} / \mathrm{animal})$ under isofluorane anesthesia. The control group received the same dose of surfactant 14 days after saline instillation. The animals were sacrificed 21 days after the induction of lung fibrosis.

Experimental groups. The animals were randomly distributed into eight experimental groups ( $n=8$ in each group):

Control: Saline instillation. 
Control + ATII Transplantation (C+ATII): Saline instillation + alveolar type II cell transplantation.

Control + AM Transplantation $(\mathrm{C}+\mathrm{AM})$ : Saline instillation + alveolar macrophage transplantation.

Control + Surfactant $(\mathrm{C}+\mathrm{SP}):$ Saline instillation + surfactant administration.

BLM: Bleomycin instillation.

BLM + ATII: Bleomycin instillation + alveolar type II cell transplantation.

BLM + AM: Bleomycin instillation + alveolar macrophage transplantation.

BLM + SP: Bleomycin instillation + surfactant administration.

For details on the hidroxyproline content, real-time PCR, Western blot studies, histology and immunostaining studies, see the online Data Supplement.

Statistical analysis. Data are expressed as mean \pm SEM values with $95 \%$ confidence intervals $(\mathrm{Cl})$. Statistical analysis was carried out by analysis of variance (ANOVA) followed by appropriate post hoc tests, including the Newman-Keuls test when differences were significant (GraphPad Software Inc, USA). A p value of $<0.05$ was considered significant.

\section{Results}

At the end of cell isolation, purity of the ATII cells was $86 \pm 6 \%$, as shown by positive staining with alkaline phosphatase or with the immunohistochemstry for p180 (Fig 1A, 1C). Results of real-time PCR showed a higher expression of SP-B and SP-C in the isolated ATII compared to the expression of these two surfactant proteins in the isolated AM (Fig 1D). Finally, the percentage of $\mathrm{p}-180$ positive cells was determined by flow cytometry in three independent experiments. The purity of isolated ATII measured by flow cytometry was 81.56 $\pm 3.26 \%$ (Fig. 1E).

BLM instillation caused a marked loss in body weight, which was normally recovered later on. The animals subjected to ATII transplantation gained weight more quickly than those in 
the BLM, BLM+AM and BLM+SP groups (Fig. 2A). This indicates that ATII transplantation was the only therapy that enhanced the animals' health. Moreover, BLM caused an increase in lung weight, owing to the inflammatory and fibrotic component (7). Lung weight significantly decreased only in the ATII transplanted group after BLM instillation, compared with the BLM, BLM+AM and BLM+SP groups (Fig. 2B). The amount of hydroxyproline - a modified amino acid specific to collagen - was assessed to determine how ATII transplantation could alter the course of BLM-induced lung injury. BLM, BLM+AM and BLM+SP lungs showed a significant increase in the amount of lung hydroxyproline when compared with saline control groups (Fig. 2C). In contrast, hydroxyproline levels were significantly reduced in the BLM+ATII transplanted group (Fig. 2C). These results confirm that ATII transplantation induces a reduction in collagen deposition and, consequently, in the fibrotic response.

Since the control groups were found to present equivalent data (data not shown) for histology, immunofluorescence, mRNA and Western blot, the results are matched in a single control group for graphic tractability.

The engraftment of the transplanted cells in the recipient animals was assessed by fluorescent microscopy. In the BLM + AM group only a few AM red PKH26 positives were observed in some areas of the lung sections (Fig. $3 A$ ). In contrast, in the BLM+ATII numerous ATII red $\mathrm{PKH} 26$ positives were observed in the transplanted animals, mainly in areas of fibrosis (Fig. 3A). The percentage of engrafted cells in the transplanted animals after one week of the infusion was $14 \pm 9 \%$.

To confirm the presence of fibrotic lesions, lung serial sections were stained with Masson trichrome and examined by light microscopy (Fig. 3B). Lung tissue sections from control rats showed no evidence of inflammation or epithelial damage but, as expected, lung tissue sections from rats with BLM-induced fibrosis showed marked peribronchiolar and interstitial infiltration, with inflammatory cells, extensive cellular thickening of interalveolar septa, interstitial edema, an increase in interstitial cells with a fibroblastic appearance and an excess of collagen deposition (Fig. 3B). Similar results were obtained in the lung tissues from 
the $B L M+A M$ and BLM+SP groups (Fig. 3B). However, although multifocal parenchymal lesions were still present in the BLM+ATII group, the organized fibroblast foci were smaller and considerably fewer than those observed in the BLM, BLM+AM and BLM+SP groups (Fig. 3B). Moreover, the reduction in parenchymal lesions was evidenced by large areas of undamaged tissue with normal alveolar architecture (Fig. 3B).

To determine one of the mechanisms involved in the beneficial effect of ATII transplantation, we studied the changes in the expression and release of pulmonary surfactant proteins (Fig. 4 and 5). Quantitative real-time PCR analysis (Fig. 4) revealed a significant decrease in the expression of all the pulmonary surfactant proteins in the BLM group, compared to the control group. ATII transplantation was the only therapy that showed a significant increase in the expression of all the pulmonary surfactant proteins, compared with the BLM group (Fig. 4). Since macrophage transplantation and surfactant administration did not induce changes in the expression of the surfactant proteins, its quantification was determined only in the $B L M+A T I I$ group. Alterations in protein expression corresponding to mRNA changes were assessed by Western blot analysis for SP-B, SP-C and SP-D. Surfactant protein levels were significantly decreased in the BLM group, compared with the control group (Fig. 5). In contrast, the levels of SP-B, SP-C and SP-D were significantly increased in the BLM+ATII group compared to the BLM group, although SP-C and SP-D did not achieve the control levels, while SP-B was increased to levels above those of the control group, albeit without any significant difference (Fig. 5). All in all, these results indicate the re-establishment of pulmonary surfactant protein levels.

To further examine the increase in the surfactant levels caused by ATII transplantation in BLM-induced pulmonary fibrosis, lung serial sections were immunostained, using an antibody anti-p180 lamellar body (Fig. 6), and then examined by fluorescent microscopy. Lung tissue sections from BLM, BLM+AM and BLM+SP showed only a weak staining for the anti-p180 lamellar body (Fig. 6). In contrast, the BLM+ATII group was once again the only group to show a marked increase in positive cells for the p180 lamellar body. Furthermore, some of the positive anti-p180 cells were donor cells as they were also positives for the red 
PKH26 marker (Fig. 6); in addition, some of these cells were located in the corner of the alveolus, the usual site of ATII (Fig 6), indicating that after ATII transplantation takes place the repopulation of the pulmonary epithelium and also the restoration of the pulmonary architecture.

\section{Discussion}

Previous studies performed by our group have demonstrated that ATII transplantation produces several beneficial effects in an animal model of BLM-induced lung fibrosis, leading ultimately to the reversal of disease (7). Since ATII are the cells exclusively responsible for the synthesis and release of some pulmonary surfactant proteins and are specifically involved in the regulation of the surfactant pool, we hypothesized that depletion of ATII during pulmonary fibrosis compromises the capacity to regulate normal levels of pulmonary surfactant, further amplifying the fibrogenic process.

The present study demonstrates that some of the beneficial effects observed after ATII transplantation may be explained by the restoration of surfactant levels. Furthermore, ATII transplantation was able to increase the levels of all four surfactant proteins. In contrast, neither AM transplantation nor exogenous surfactant administration led to any recovery in the expression of surfactant proteins, or in body weight, nor a decrease in collagen deposition or an improvement in parenchymal lesions. These results confirm that ATII transplantation has a specific role in the resolution of disrupted alveolar surfaces, partly by inducing the restoration of surfactant levels.

It is known that ATII death is a key component in the progression of pulmonary fibrosis (21). ATII synthesizes all four surfactant proteins, and SP-C is the only surfactant protein expressed exclusively by ATII in the mature lung. SP-A, SP-B and SP-D may also be expressed by other airway cells, such as Clara cells and submucosal cells (4-8). Although fibrosis extend into alveolar spaces and induce epithelial cells damage, other airway cells capable of maintaining the expression and synthesis of SP-A, SP-B and SP-D could still remain unaffected. However, in our study both the mRNA and protein of all four surfactant 
proteins were significantly reduced in lung homogenates of animals treated with BLM, indicating a broad extension of fibrotic areas in this animal model. In this sense, it is important to point out that surfactant synthesis, internalization, reutilization and secretion comprise a highly regulated system that maintains appropriate levels of pulmonary surfactant throughout life. This process is essential for proper respiratory function, (22-24). ATII are the specific cells that recycle surfactant proteins and phospholipids from the alveolar space incorporating them directly into their secretory pathway. Therefore, any additional alterations to surfactant turnover caused by ATII death may lead to a marked loss of surface tensionlowering capacity, inducing changes in the biophysical and immunological surfactant function. Furthermore, epithelial and endothelial damage at the alveolo-capillary junction in pulmonary fibrosis results in plasma leakage, accumulation of fibrin, inflammatory cell and necrotic cell debris, while the formation of hyaline membranes causes alterations resulting from the inhibition or degradation of surfactants (13). This phenomenon has been observed in IPF patients, where neutrophil infiltration is capable of degrading surfactant proteins (25). Altogether, these factors indicate that alterations in the surfactant system during the development of pulmonary fibrosis may amplify the fibrogenic processes.

The most important result of this study is that only the ATII transplantation induced the restoration of the surfactant protein pool. In contrast, neither macrophage transplantation nor surfactant administration were able to recover the expression levels of any surfactant proteins after BLM administration. Furthermore, ATII transplantation was able to increase the release of all surfactant proteins. This recovery of surfactant protein suggests not only the expression and release of new protein but also the reestablishment of the recycling capacity intrinsic to healthy transplanted ATII. Nevertheless, we observed that positive cells for SP-C were not only transplanted ATII but also own ATII cells, indicating that after ATII transplantation takes place the recovery of the own pulmonary epithelium cell functionality. This means that the restoration of the surfactant system could be attributed not only to the new donor cells but also to the recruitment of own epithelial cells thus amplifying the beneficial effect of ATII transplantation. The functional surfactant synthesized and released 
will keep the alveoli clear of liquid, while also maintaining a thin fluid film that will enhance breathing capacity. These findings were only induced in the ATII transplantation group. In contrast, surfactant administration was not able to reestablish the surfactant system, increasing the importance of healthy ATII in opening up collapsed but still functional alveoli. In this regard, the immunofluorescence studies confirm all these results, as the lung of animals transplanted with ATII showed extensive restoration of the pulmonary architecture, together with a clear increase in the number of surfactant positive cells.

In conclusion, this study shows that one of the mechanisms involved in the beneficial effect of ATII transplantation is the restoration of the lung surfactant protein levels, released by themselves or trough the recovery of the own pulmonary epithelium cell functionality needed for proper respiratory function. 
Acknowledgements: We thank Luis Ignacio Sanchez and Valeria Sirenko for technical assistance. We also thank the Citomics unit of the Institut d'Investigacions Biomèdiques August Pi i Sunyer (IDIBAPS) for the technical help.

Contributor statement: Conception and design: RGP, GGJ, and ASM; Analysis and interpretation: RGP, GGJ, AX, VIP and ASM; Drafting the manuscript for important intellectual content: RGP, VIP and AMS.

This article has an online data supplement. 


\section{References}

1. Raghu G, Collard HR, Egan JJ, et al. ATS/ERS/JRS/ALAT Committee on Idiopathic Pulmonary Fibrosis. An official ATS/ERS/JRS/ALAT statement: idiopathic pulmonary fibrosis: evidence-based guidelines for diagnosis and management. Am J Respir Crit Care Med. 2011:183:788-824.

2. Noble PW, Albera C, Bradford WZ, et al. Pirfenidone in patients with idiopathic pulmonary fibrosis (CAPACITY): two randomised trials. Lancet. 2011:377:1760-9.

3. Richeldi L, Costabel U, Selman M, et al. Efficacy of a tyrosine kinase inhibitor in idiopathic pulmonary fibrosis. N Engl J Med. 2011:365:1079-87.

4. Sisson TH, Mendez M, Choi K, et al. Targeted injury of type II alveolar epithelial cells induces pulmonary fibrosis.Am J Respir Crit Care Med. 2010:181:254-63.

5. Korfei M, Ruppert C, Mahavadi P, et al. Epithelial endoplasmic reticulum stress and apoptosis in sporadic idiopathic pulmonary fibrosis. Am J Respir Crit Care Med. 2008:178:838-46.

6. Thannickal VJ, Toews GB, White ES, et al. Mechanisms of pulmonary fibrosis. Annu Rev Med. 2004.55:395-417.

7. Serrano-Mollar A, Nacher M, Gay-Jordi G, et al. Intratracheal transplantation of alveolar type II cells reverses bleomycin-induced lung fibrosis. Am J Respir Crit Care Med. 2007:176:1261-8.

8. Zoz DF, Lawson WE, Blackwell TS. Idiopathic pulmonary fibrosis: a disorder of epithelial cell dysfunction. Am J Med Sci. 2011:341:435-8.

9. Gower WA, Nogee LM. Surfactant dysfunction. Paediatr Respir Rev. 2011;12:223-9.

10. Burkhardt A. Alveolitis and collapse in the pathogenesis of pulmonary fibrosis. Am Rev Respir Dis. 1989:140:513-24.

11. Garcia CK. Idiopathic pulmonary fibrosis: update on genetic discoveries. Proc Am Thorac Soc. 2011:8:158-62.

12. Nogee LM. Genetic mechanisms of surfactant deficiency. Biol Neonate. 2004:85:3148. 
13. Günther A, Schmidt R, Nix F, et al. Surfactant abnormalities in idiopathic pulmonary fibrosis, hypersensitivity pneumonitis and sarcoidosis. .Eur Respir J. 1999:14:565-73.

14. Lawson WE, Grant SW, Ambrosini V, et al. Genetic mutations in surfactant protein C are a rare cause of sporadic cases of IPF. Thorax. 2004:59:977-80.

15. Chibbar R, Shih F, Baga M, Torlakovic E, Ramlall K, Skomro R, Cockcroft DW, Lemire EG. Nonspecific interstitial pneumonia and usual interstitial pneumonia with mutation in surfactant protein $\mathrm{C}$ in familial pulmonary fibrosis. Mod Pathol. 2004:17:973-80.

16. Casals $\mathrm{C}$, Johansson $\mathrm{H}$, Saenz A, et al. C-terminal, endoplasmic reticulum-lumenal domain of prosurfactant protein $\mathrm{C}$ - structural features and membrane interactions. FEBS J. 2008:275:536-47.

17. Hardie WD, Hagood JS, Dave V, et al. Signaling pathways in the epithelial origins of pulmonary fibrosis. Cell Cycle. 2010 Jul 15;9(14):2769-76.

18. Aono Y, Ledford JG, Mukherjee S, et al. Surfactant protein-D regulates effector cell function and fibrotic lung remodeling in response to bleomycin injury. Am J Respir Crit Care Med. 2012 Mar 1;185(5):525-36.

19. Lawson WE, Cheng DS, Degryse AL, et al. Endoplasmic reticulum stress enhances fibrotic remodeling in the lungs. Proc Natl Acad Sci U S A. 2011:108:10562-7.

20. Lawson WE, Crossno PF, Polosukhin VV, et al. Endoplasmic reticulum stress in alveolar epithelial cells is prominent in IPF: association with altered surfactant protein processing and herpesvirus infection Am J Physiol Lung Cell Mol Physiol. 2008:294:L1119-26.

21. Griese M. Pulmonary surfactant in health and human lung diseases: state of the art. Eur Respir J. 1999:13:1455-76.

22. Baritussio A, Pettenazzo A, Benevento $M$, et al. Surfactant protein $C$ is recycled from the alveoli to the lamellar bodies. Am J Physiol Lung Cell Mol Physiol 1992:263:L607-L611. 
23. Breslin JS, Weaver TE. Binding, uptake, and localization of surfactant protein B in isolated rat alveolar type II cells. Am J Physiol Lung Cell Mol Physiol 1992: 262:L699_ L707

24. Ryan RM, Morris RE, Rice WR, et al. Binding and uptake of pulmonary surfactant protein (SP-A) by pulmonary type II epithelial cells. J Histochem Cytochem 1989:37:429-440

25. Pison U, Tam EK, Caughey GH, et al. Proteolytic inactivation of dog lung surfactantassociated proteins by neutrophil elastase. Biochim Biophys Acta. 1989:992:251-7. 


\section{Legends}

A

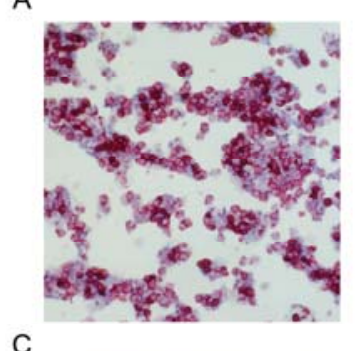

C

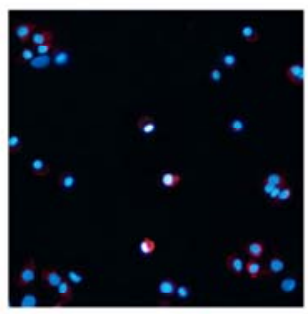

B
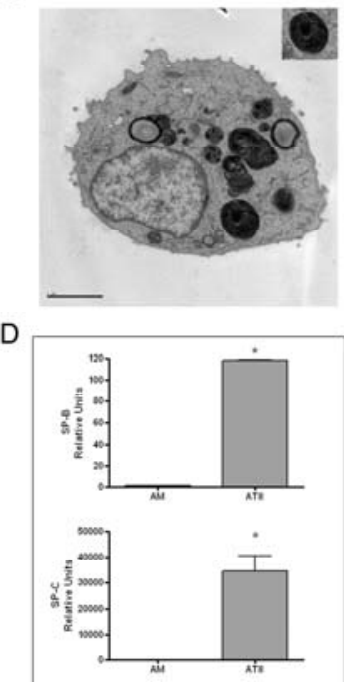

$\mathrm{E}$

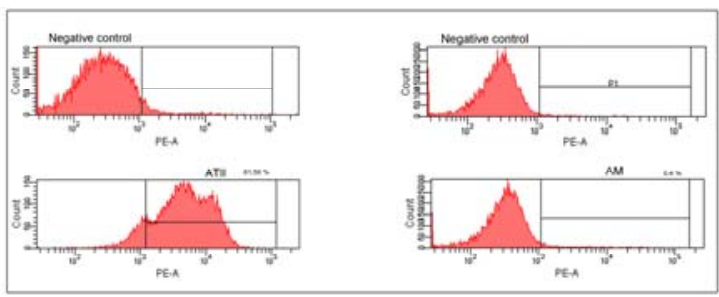

$\mathrm{F}$

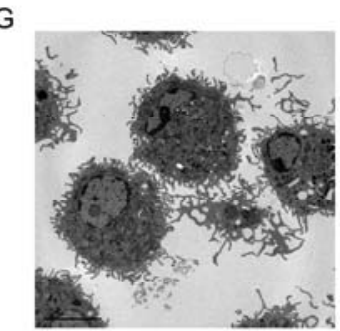

Figure 1. Purity of isolated donor cells. (A) In red, positive ATII cells for alkaline phosphatase after cytospin. Magnification x 200. (B) Transmission electron micrograph of an ATIl cell showing lamellar bodies (see detail in the upper right corner). (C) Immunochemistry for ATII cells against p180 protein. Positive cells are labelled in red. Magnification $\times 400$ (D) Realtime PCR for SP-B and SP-C of isolated ATII and AM. (E) Flow cytometry analysis of p180 in isolated ATII and isolated AM. Cells were immunostained with PE-labelled anti p180 antibody. Flow cytometry analysis of p180 in isolated AM were used as a control for the 
analysis of isolated ATII purity. Cells were immunostained with PE-labelled anti SP-C antibody. (F) Diff-Quick stain of AM after cytospin . Magnification x 200. (G) Transmission electron micrograph of AM.
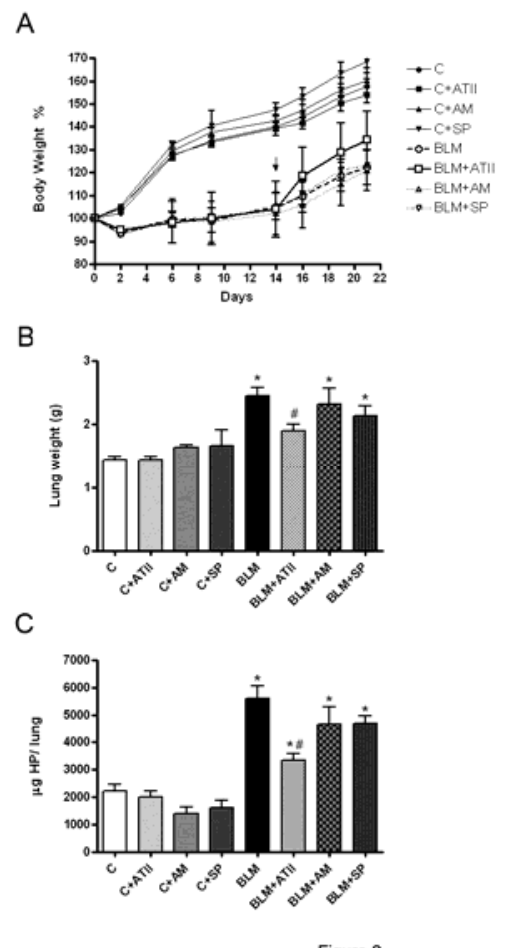

Figure 2

Figure 2. Biomarkers of lung fibrosis progression (A) Curves of animal body weight over time. On day 0 , the animals received bleomycin (BLM). On day 14 , animals were treated with ATII,AM or surfactant (Arrowhead). (B) Lung weight at the end of the experiment (21 days) in all the experimental groups. (C) Lung hydroxyproline levels at the end of the experiment (21 days) in all the experimental groups. Data are means \pm SEM ( $n=8$ animals per group) ( ${ }^{*} p<0.051$ vs control groups, \# $p<0.05$ vs BLM group). 

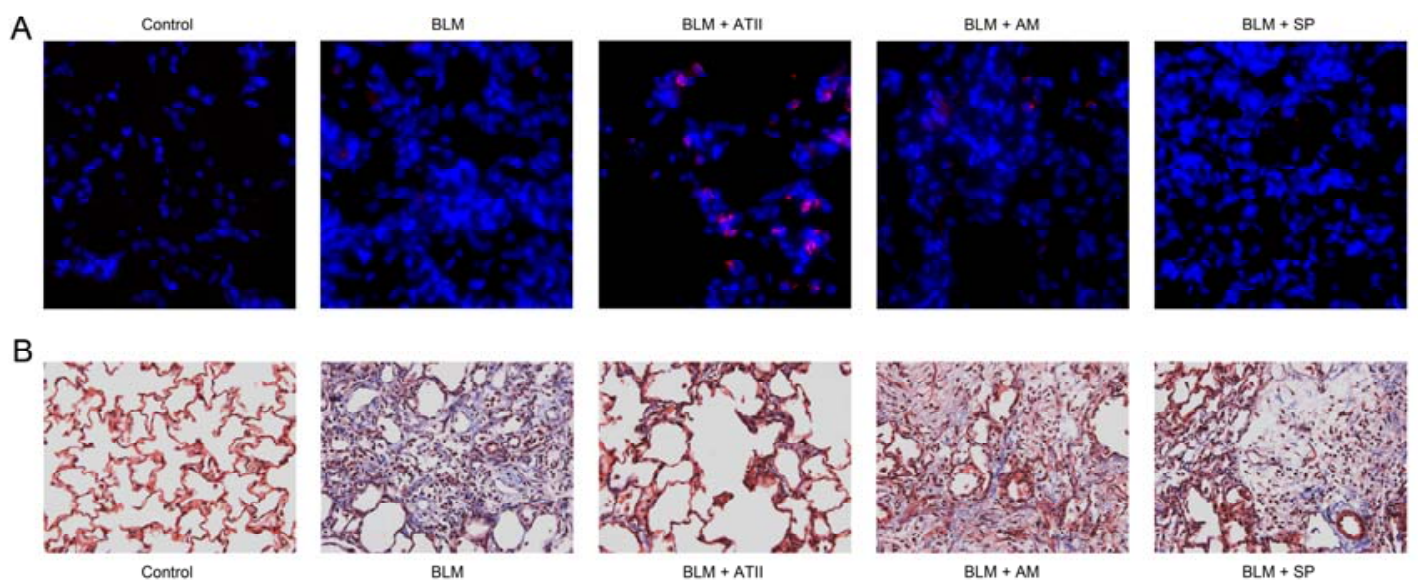

BLM + ATII

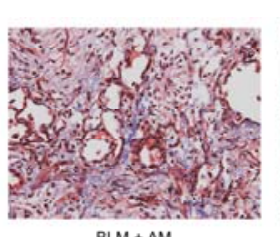

BLM + AM

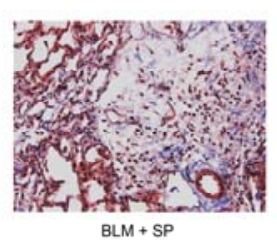

$\mathrm{BLM}+\mathrm{SP}$

Figure 3. Lung histology in experimental groups (A) Fluorescence microscopy of ATII and AM engraftment after 21 days of cell infusion. For tracking cell purposes, donor cells were labelled in red with PKH26 (group BLM+ATII and group BLM+AM). Nuclei were stained in blue with DAPI. Magnification x 400. (B) Bright field microscopy photomicrographs of lung histopathology at the end of the experiment (21 days) in all the experimental groups. Lung sections were stained with Masson trichrome. ATII transplantation is the only therapy that is able to ameliorate the inflammatory and pulmonary lesions. The presence of interstitial collagen (blue staining) was also diminished only in the BLM+ATII group. Magnification $x$ 200. 


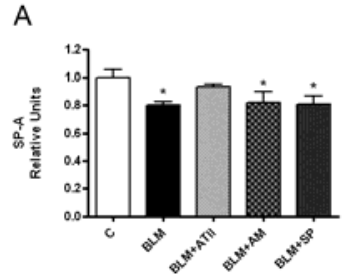

C

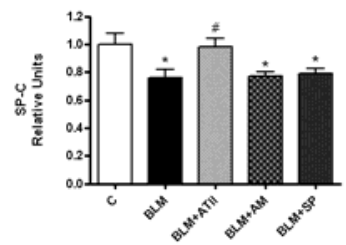

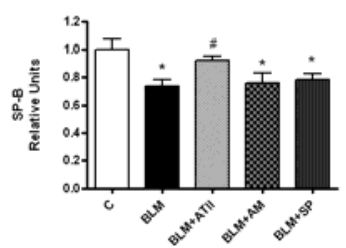

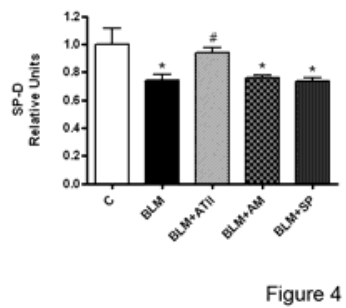

Figure 4. Gene expression of four surfactant proteins in lung tissue. (A) SP-A, (B) SP-B, (C) SP-C and (D) SP-D at the end of the experiment (21 days) in all groups. Results are obtained according to the $\Delta \mathrm{Ct}$ method related to GAPDH. Bars represent the fold change \pm SEM with respect control group ( $\mathrm{n}=8$ animals per group). ${ }^{*} \mathrm{p}<0.0501$ vs control groups, \# $p<0.05$ vs BLM group. 
A

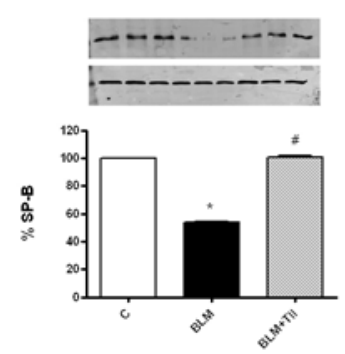

$B$
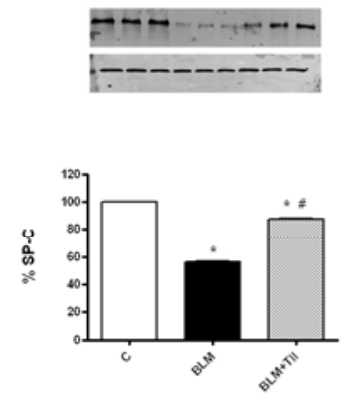

C

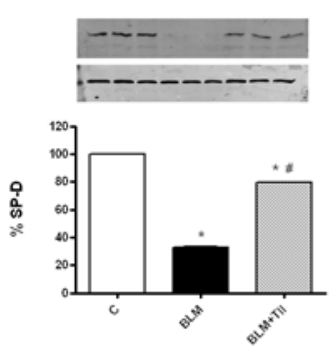

Figure 5

Figure 5. Protein levels of three pulmonary surfactant proteins. (A) SP-B, (B) SP-C and (D) SP-D from lung tissue at the end of the experiment (21 days) in all the experimental groups. Protein level was normalized for tubulin expression. Data are mean \pm SEM $(n=8$ animals per group). ${ }^{*} p<0.05$ vs control groups, $\# p<0.05$ vs BLM group.
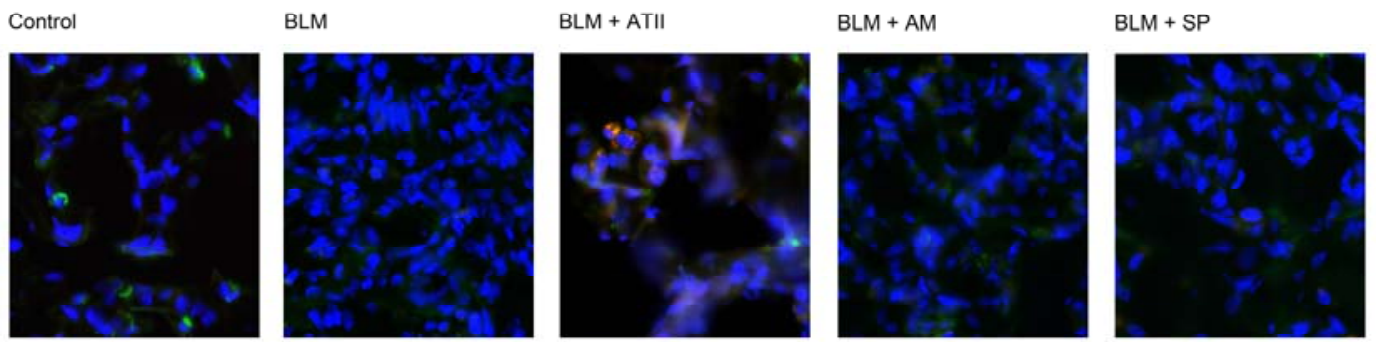

Figure 6

Figure 6. Immunofluorescence for p180 lamellar body in lung tissue in all the experimental groups at the end of the experiment ( 21 days). In green is shown positive cells for p180 
lamellar body. ATII donor cells were labelled in red with PKH26. Colocalization of p180 and PKH26 is shown in yellow only observed in BLM+ATII transplanted group. Nuclei were stained in blue with DAPI. Magnification x 640. 


\section{Online Data Supplement}

This appendix has been provided by the authors to give readers additional information about their work.

Supplement to: ALVEOLAR TYPE II CELL TRANSPLANTATION RESTORES PULMONARY SURFACTANT PROTEIN LEVELS IN LUNG FIBROSIS

Raquel Guillamat-Prats ${ }^{1,2}$, Gemma Gay-Jordi ${ }^{1,2}$, Antoni Xaubet ${ }^{2,3}$, Victor I Peinado ${ }^{2,3}$, Anna Serrano-Mollar ${ }^{1,2}$ 


\section{SUPPLEMENTAL METHODS}

Isolation of Alveolar Type II Cells. Fresh alveolar type II cells (ATII) were isolated from healthy donor animals. To isolate ATII, the lungs were removed from the animal and lavaged with $5 \times 10 \mathrm{ml}$ saline. The lungs were digested by filling with $0.25 \%$ trypsin dissolved in saline (100 ml) (T8003, Sigma, Missouri, USA) and suspended in $0.9 \% \mathrm{NaCl}$ at $37^{\circ} \mathrm{C}$ for $30 \mathrm{~min}$, with the trypsin constantly topped up to expand the parenchyma for $30 \mathrm{~min}$, suspended in a saline solution at $37^{\circ} \mathrm{C}$. Following digestion, the lungs were chopped into $1-2 \mathrm{~mm}^{2}$ cubes, treated with $75 \mathrm{U} / \mathrm{ml}$ DNase dissolved in saline and filtered through nylon meshes ranging from 150 to $30 \mu \mathrm{m}$. The resulting cell suspension was centrifuged $\left(250 \times \mathrm{g}, 20 \mathrm{~min}\right.$ at $\left.10^{\circ} \mathrm{C}\right)$ through a sterile Percoll gradient and the ATII rich band was removed. A second DNase treatment of $20 \mathrm{U} / \mathrm{ml}$ was administered and the cells were recovered as a pellet by centrifugation at $250 \times \mathrm{g}$ for $20 \mathrm{~min}$. These cells were resuspended in $5 \mathrm{ml} \mathrm{DCCM} 1$ (Biological Industries, Kibbutz Beit Haemek, Israel) completed with a 2\% (w/v) L- Glutamine and subjected to differential attachment on a plastic Petri dish. Non-adherent ATII were collected after $2 \mathrm{~h}$ and counted to establish the final cell yield of freshly purified cells.

The ATII viability was assessed by Tripan Blue (Sigma, Missouri, USA), showing $>95 \%$ viability.

Cell yield and purity of Alveolar Type II Cells isolation. Cell yield and purity were assessed by different techniques: by alkaline phosphatase staining kit (Sigma, Missouri, USA), by immunochemistry, by real-time PCR and by flow cytometry.

To perform the phospatase alkaline kit and the immunochemistry assay isolated ATII were cytocentrifuged (Cytospin 3 centrifuge Shandon Scientific Ltd, Grupo Taper, Spain), allowed to dry in air for $15 \mathrm{~min}$ at RT. The phosphatase staining was conducted according to the manufacturer's protocol for alkaline phosphatase.

Immunochemistry analysis were done in ATII cytospins, the cells were labelled using an antibody against p180 lamellar body (Abcam, UK), specific to surfactant lamellar bodies, and 
revealed with a secondary antibody anti-mouse Alexa fluor 594 (Life Technologies, Spain). The nuclei were labelled with DAPI.

Real-time PCR was performed to verify the expression of SP-B and SP-C in the isolated ATII. Isolated AM were used as a negative control. Total RNA was extracted using TRIzol reagent (Invitrogen, Carlsbad, CA, USA) and reverse-transcribed into cDNA, according to the manufacturer's instructions. Quantitative real-time PCR amplification was performed using TaqMan technology and mix buffer Universal PCR Master Mix No Amp Erase ${ }^{\circledR}$ UNG (Applied Biosystems, CA, USA). The cycle conditions were as follows: $95{ }^{\circ} \mathrm{C}, 10 \mathrm{~min}$, followed by 40 cycles of amplification $\left(95{ }^{\circ} \mathrm{C}\right.$ denaturation, $15 \mathrm{~s}$, and $60{ }^{\circ} \mathrm{C}$ combined annealing/extension for $1 \mathrm{~min})$. The primers used came from Applied Biosystems: SP-B, Rn00569225-m1 for SP-C, Rn00563557-m1 and for GAPDH. Results are expressed according to the $\Delta \mathrm{Ct}$ method related to GAPDH $(n=8)$.

Finally, the puryity of ATII isolation was analyzed by flow cytometry. The flow cytometry assays were performed in triplicate. For each assay, $1 \times 10^{6}$ cells were analyzed. Flow cytometry analysis was performed with a cytometer (FACSCanto II, BD Pharmingen, San Jose, CA). The data were analyzed with a specific software (FACSDiva ${ }^{\mathrm{TM}}$, BD Pharmingen). Cells were incubated with irrelevant immunoglobulins IgG (Sigma-Aldrich) to block Fc receptors for nonspecific binding and then resuspended in $1000 \mu$ of a solution of permeabilization (saponine $0.05 \%$ in PBS) and incubated for 10 min at $4{ }^{\circ} \mathrm{C}$. The cells then were incubated with the p180 lamellar body (Abcam, UK) primary antibody (dilution 1/100) in the dark for $15 \mathrm{~min}$ at $4{ }^{\circ} \mathrm{C}$. The cellular pellet then was resuspended and incubated in the dark for 15 min at $4{ }^{\circ} \mathrm{C}$ with the secondary antibody conjugated with PE (dilution 1/50) in PBS. The cells were washed $(2 \times 5 \mathrm{~min} \times 1200 \mathrm{rpm})$ with $1 \mathrm{ml}$ of PBS. The cells were then fixed with $0.01 \%$ para-formaldehyde $+0.1 \%$ of sodium azide in PBS and incubated in the dark for $15 \mathrm{~min}$, washed $(2 \times 5 \mathrm{~min} \times 1200 \mathrm{rpm})$ with $1 \mathrm{ml}$ of PBS $+0.1 \%$ sodium azide and resuspended with PBS $+0.1 \%$ sodium azide until the analysis. Alveolar macrophages (AM) were labelled with the antibody p180 lamellar body and used as a negative control of isolated ATII purity. 
Isolation of Alveolar Macrophage. Fresh AM were isolated from healthy donor animals by performing a bronchoalveolar lavage. Bronchoalveolar lavage was undertaken by washing the lung five times with $10 \mathrm{ml}$ saline aliquots via a tracheal cannula and centrifuging $(300 \times \mathrm{g}$, $20 \mathrm{~min}, 4^{\circ} \mathrm{C}$ ). The AM viability after isolation was assessed by Trypan Blue and proved to be $>95 \%$.

Transmission electron microscopy. The ATII and AM characterization was evaluated by transmission electron microscopy. The cells were fixed in $2.5 \%$ glutaraldehyde in $0.1 \mathrm{~mol} / \mathrm{l}$ phosphate buffer $\left(\mathrm{pH} \mathrm{7.4)}\right.$ for at least $2 \mathrm{~h}$ at $4{ }^{\circ} \mathrm{C}$. The cells were secondarily fixed in $1 \%$ osmium tetroxide and $0.8 \%$ potassium ferrocyanide for $1 \mathrm{~h}$ at $4{ }^{\circ} \mathrm{C}$. After 3 washes with cold double distilled water, the sample was dehydrated with an ascending concentration of acetone $(30 \%, 50 \%, 70 \%, 95 \%$, and $100 \%)$, and three changes of $100 \%$ acetone. They were then embedded in Spurr resin and polymerized at $60{ }^{\circ} \mathrm{C}$. The embedded blocks were sectioned using a diamond knife (Diatome) on a Leica Ultracut UCT (Leica Microsystems, Deerfield, IL). Ultrathin sections were placed on copper grids and stained with ranyl acetate and lead citrate before examination under a JEM 1010 (Jeol Ltd., Tokio, Japan) equipped with a Gatan/BioScan digital camera (Gatan Inc., Pleasanton, CA).

Cell Engraftment. After cell isolation the ATII and the AM were labeled by the PKH26 Red Fluorescent Cell Linker Kit (Sigma, Missouri, USA) following the manufacturer's protocol, and then transplanted to the animals. To evaluate cell engraftment, the lungs were collected, frozen and embedded in OCT (Jung, Japan). Eight $\mu \mathrm{m}$ sections were examined under fluorescent microscopy. The nuclei were stained with DAPI. The percentage of engrafted cells was evaluated by counting positive $\mathrm{PKH} 26$ cells over the total cell number on 15 randomly selected fields by two blinded different observers.

Hidroxyproline content. Lung hydroxyproline content was measured as an indicator of collagen deposition, following the method outlined by Woessner (E1). Samples were homogenized and then hydrolyzed in $6 \mathrm{M} \mathrm{HCl}$, and the hydrolysate was then neutralized with 
$2.5 \mathrm{M} \mathrm{NaOH}$. Hydroxyproline in the hydrolysate was assessed colorimetrically at $550 \mathrm{~nm}$ with p-dimethylaminobenzaldehyde. The results are expressed as $\mu \mathrm{g}$ of hydroxyproline per lung. RNA isolation and real-time PCR analysis. Total RNA was extracted from lung tissue using TRIzol reagent (Invitrogen, Carlsbad, CA, USA) and reverse-transcribed into cDNA, according to the manufacturer's instructions. Quantitative real-time PCR amplification was performed using TaqMan technology and mix buffer Universal PCR Master Mix No Amp Erase ${ }^{\circledR}$ UNG (Applied Biosystems, CA, USA). The cycle conditions were as follows: $95{ }^{\circ} \mathrm{C}$, $10 \mathrm{~min}$, followed by 40 cycles of amplification $\left(95^{\circ} \mathrm{C}\right.$ denaturation, $15 \mathrm{~s}$, and $60{ }^{\circ} \mathrm{C}$ combined annealing/extension for $1 \mathrm{~min})$. The primers used came from Applied Biosystems: Rn00824545-m1 for SP-A, Rn00593742-m1 for SP-B, Rn00569225-m1 for SP-C, Rn00563557-m1 for SP-D and Rn-99999916-s1 for GAPDH. Results are expressed according to the $\Delta \mathrm{Ct}$ method related to GAPDH ( $\mathrm{n}=8$ in each group).

SDS-PAGE and Western blot. Protein samples were extracted using Nonidet P-40 buffer. SDS-PAGE was performed on 5\%-13\% acrylamide gels. Proteins were electrotransferred to nitrocellulose membrane and probed with primary antibodies. The antibodies used included mouse anti-SP-B (dilution1/2000) (Acris Antibodies, Germany), rabit anti-SP-C (dilution 1/1000) (Santa Cruz Biotechnology, USA), anti-SP-D (dilution 1/1000) (Acris Antibodies, Germany), and mouse anti- alpha-tubulin (dilution (1/1000) (Sigma, USA), which served as a housekeeping reference. The membranes were incubated with the corresponding peroxidase-conjugated secondary antibodies, washed, and then incubated with ECL reagents (GE Healthcare Europe $\mathrm{GmbH}$; Freigburg; GE) before exposure to high performance chemiluminescence films. Gels were calibrated using Bio-Rad standard proteins (Hercules, CA) with markers covering a 7-240 kDa range.

Films were scanned by using image-editing software NIH ImageJ software for densitometric analysis of immunoreactive bands.

Immunostaining studies. For the immunofluorescence studies the lungs were collected, frozen and embedded in OCT (Jung, Japan). Eight $\mu \mathrm{m}$ tissue sections were obtained and the pulmonary surfactant proteins were assessed using an antibody against p180 lamellar body 
(Abcam, UK), specific to surfactant lamellar bodies, and revealed with a secondary antibody anti-mouse FITC (Acris, Germany). The surfactant was observed in green colour. The nuclei were stained with DAPI.

Histology. Lungs used for histology were inflated and fixed with $10 \%$ neutral buffered formalin, immersed in the fixative solution for $24 \mathrm{~h}$ and paraffin-embedded. Four $\mu \mathrm{m}$ sections were stained with Masson's trichrome to identify connective tissue and collagen deposition. 


\section{SUPPLEMENTAL REFERENCES}

1. Woessner JF Jr. The determination of hydroxyproline in tissue and protein samples containing small proportions of this imino acid. Arch. Biochem. Biophys. 1961:93: 440-447. 